\title{
Early Fault Diagnosis Technology for Bearings Based on Quantile Multiscale Permutation Entropy
}

\author{
Yufeng Long $\mathbb{D}^{1},{ }^{1}$ Xianjun Shi $\mathbb{D},,^{1}$ Qiangqiang Chen $\mathbb{D},{ }^{2}$ Zhicai Xiao $\mathbb{D},{ }^{1}$ Yufeng Qin $\mathbb{D},{ }^{1}$ \\ and Jiapeng $\operatorname{Lv} \mathbb{D}^{1}$ \\ ${ }^{1}$ Naval Aviation University, Yantai 264001, China \\ ${ }^{2}$ Naval Research Institution, Shanghai 200436, China \\ Correspondence should be addressed to Yufeng Long; ytlongyufeng007@163.com
}

Received 19 August 2021; Accepted 19 October 2021; Published 29 October 2021

Academic Editor: Marcelo Ra l Risk

Copyright (C) 2021 Yufeng Long et al. This is an open access article distributed under the Creative Commons Attribution License, which permits unrestricted use, distribution, and reproduction in any medium, provided the original work is properly cited.

Early fault diagnosis of bearings is the basis of condition-based maintenance. To overcome the difficulty of early fault diagnosis for the mechanical system, a new conception named quantile multiscale permutation entropy (QMPE) is defined, and a new feature extraction method based on QMPE is proposed. On the basis of the multiscale entropy, the multiscale permutation entropy for the gathered vibration signal of equipment is obtained, and the sample quantile is calculated, which is employed to analyze the weak change of the variation signal. The proposed method is verified with the full lifetime datasets of a certain bearing, which proves that signal features extracted by the QMPE method can not only truly express the bearing detailed condition changing from normal to fault but also duly detect the early fault of the bearing. Comparing with other methods for early fault diagnosis, the proposed method can advance the finding time of the early fault obviously.

\section{Introduction}

Rolling bearings are one of the most basic structures in a mechanical system, as well as an important part of the mechanical system. The running state of the rolling bearing is directly related to the safe and stable operation of the system [1]. From normal operation to the failure stage, rolling bearings often go through a series of different degrees of degradation. If the degradation of rolling bearings can be quantitatively evaluated during this period, equipment maintenance programs can be formulated in a targeted manner. However, in the process of rolling bearing state changes, the degree of failure is a process that continuously accumulates over time [2]. How to get the degradation state from this weak degradation process and how to extract the signal characteristics from the nonlinear and nonstationary vibration signal are necessary conditions for the early fault diagnosis of the bearing.

The selection of the characteristic parameters of the vibration signal is very important for the effective evaluation of the bearing's operating state. Typical time-domain feature parameters such as root mean square value, peak-to-peak value, kurtosis, and other features are widely used in feature extraction [3]. In the frequency-domain feature selection, there are performance characteristics such as spectral variance and spectral average [4]. In order to make effective use of these characteristic parameters, multidimensional features are usually selected to evaluate the running state of rolling bearings. Xu et al. [5] selected 12 time-frequencydomain indicators, used the local linear embedding method to reduce dimensionality, and finally obtained 7 indicators including root mean square, kurtosis index, and root square amplitude for recession prediction. Wang [6] selected 12 dimensional indicators and 6 nondimensional indicators in the time domain, while selecting 9 indicators in the frequency domain, and reduced the dimensionality through principal component analysis, so as to predict the degradation trend of rolling bearings. However, affected by many factors in the bearing degradation process, the traditional time-frequency-domain indicators are usually only sensitive to the degradation state of a specific failure mode at a specific stage, and the ability to characterize the comprehensive 
performance degradation during the life cycle of the rolling bearing is insufficient.

For rolling bearings, the complexity of vibration signals will always change along with the process of bearing performance degradation. Therefore, using entropy theory to measure the overall complexity of the signal has great advantages in performance degradation state evaluation and prediction. Zhou et al. [7] used wavelet packet singular spectrum entropy to evaluate the performance degradation of rolling bearings. Zhou et al. [8] introduced Tsallis entropy to establish a rolling bearing performance degradation evaluation method. Feng et al. [9] introduced empirical mode decomposition (EMD) and entropy. The algorithm performs bearing abnormality detection; Feng et al. [10] used the wavelet correlation filtering method to decompose and filter the rolling bearing signal and expressed the early fault characteristics of the bearing through permutation entropy. The permutation entropy algorithm can effectively reflect the small changes in time series data and has good applications in the fields of medicine and biology [11].

The permutation entropy algorithm has good performance at a single feature scale and can effectively detect the randomness and dynamic mutation of time series. In order to extend the performance of the single-scale entropy algorithm, Wang et al. [12] introduced multiscale entropy to predict the remaining life of the bearing. Zhang et al. [13] introduced multiscale entropy partial mean information to evaluate the degree of bearing failure, which improved the performance of fault diagnosis to a certain extent. Based on the multiscale permutation entropy, this paper defines a new quantitative indicator of failure degree-quantile multiscale permutation entropy (QMPE). QMPE extracts sample quantiles of permutation entropy values at multiple scales, which effectively reflects the aggregation characteristics of data at a certain quantile point, thereby deeply extracting the characteristic state information of the full life cycle of rolling bearings, which can be effectively used for abnormal detection and early fault diagnosis. Studies have shown that this method can effectively express fault characteristic information and significantly advance the discovery time of early bearing faults.

\section{Multiscale Permutation Entropy}

2.1. Permutation Entropy. The principle of the permutation entropy algorithm is as follows: for a time series $\{x(i), i=1,2, \ldots, N\}$ of length $N$, reconstruct the phase space of $x(i)[14]$ :

$$
\left\{\begin{array}{c}
\mathbf{X}_{1}=\{x(1), x(1+\tau), \ldots, x[1+(m-1) \tau]\}, \\
\mathbf{X}_{j}=\{x(j), x(j+\tau), \ldots, x[j+(m-1) \tau]\}, \\
\vdots \\
\mathbf{X}_{k}=\{x(k), x(k+\tau), \ldots, x[k+(m-1) \tau]\},
\end{array}\right.
$$

where $m$ is the embedding dimension, $\tau$ is the delay time, and $k=N-(m-1) \tau$ is the number of reconstruction vectors.
The elements of $\mathbf{X}_{j}$ can be rearranged in an increasing order as

$$
x\left[i+\left(j_{1}-1\right) \tau\right] \leq \cdots \leq x\left[i+\left(j_{m}-1\right) \tau\right],
$$

where $j_{1}, j_{2}, \ldots, j_{m}$ is the index of each element in the column of the phase space before sorting.

If two elements in $\mathbf{X}_{j}$ are equal, they are arranged in the original order. Through the analysis of the time series after phase space reconstruction, for any $\mathbf{X}_{j}$, the corresponding symbol sequence $\mathbf{S}_{l}=\left\{j_{1}, j_{2}, \ldots, j_{m}\right\}$ can be obtained, where $l=1,2, \ldots, k$ and $k \leq m !$. The permutation entropy can be defined as [15]

$$
H_{p}(m)=-\sum_{j=1}^{k} P_{j} \ln \left(P_{j}\right)
$$

where $P_{j}$ is the probability of the symbol sequence, and $\sum_{j=1}^{k} P_{j}=1$.

If $P_{j}=1 / m !, H_{p}(m)=\ln (m !)$, and the value is the largest. Standardize $H_{p}(m)$ to get

$$
H_{p}=\frac{H_{p}(m)}{\ln (m !)} .
$$

At this time, the value of $H_{p}$ is $[0,1]$, which can reflect the complexity of the time series. The larger $H_{p}$, the higher the complexity of the sequence.

2.2. Multiscale Permutation Entropy. Multiscale permutation entropy is defined as permutation entropy under different scales, and the calculation method is as follows:

(1) Given the time series $\{x(i), i=1,2, \ldots, N\}$, and coarse-grain it to obtain the coarse-grained sequence $\left\{y_{j}^{(\tau)}\right\}$ :

$$
y_{j}^{(\tau)}=\frac{1}{\tau} \sum_{i=(j-1) \tau+1}^{j \tau} x_{i},
$$

where $j=1,2, \ldots,[N / \tau],[N / \tau]$ means rounding $N / \tau$; $\tau$ is the scale factor; when $\tau=1$, the coarsegrained sequence is the original sequence, and the multiscale permutation entropy is the original permutation entropy.

(2) Calculate the permutation entropy of each coarsegrained sequence and describe it as a function of the scale factor. The permutation entropy at multiple scales obtained is the multiscale permutation entropy. The value of the scale factor affects the multiscale permutation entropy analysis to a certain extent.

\section{QMPE Feature Extraction Method}

By setting the scale factor $\tau$, the permutation entropy values of $\tau$ different scales can be obtained. In the process of analyzing the failure of rolling bearings, Zheng et al. [16] selected the first four multiscale array entropy values as the eigenvectors (i.e., $\tau=1,2,3$, and 4); Ren et al. [17] completed the online 
monitoring of milling chatter by selecting two multiscale permutation entropy values with $\tau=1$ and $\tau=4$ as the feature vector; in [18], in monitoring the abnormal state of EEG signals, it is also based on different multiscale permutation entropies under different scale factors $\tau$ of epilepsy patients and normal patients to distinguish the disease conditions. In the study of failure mode recognition, it is only necessary to distinguish the difference of the failure under different failure characteristics. However, in the prediction of the degradation trend of rolling bearings and the performance degradation assessment, the degradation characteristics are required to have a strong follow-up response to the changes in the performance degradation of rolling bearings. At this time, the multiscale permutation entropy value under a certain scale factor $\tau$ alone is not enough to fully reflect the performance degradation trend. However, when selecting the entropy value of the multiscale permutation under a certain scale factor $\tau$ as the feature parameter, there are some information redundancy and mutual conflict problems. Therefore, it is necessary to deeply extract relevant information from the multiscale permutation entropy at different scales and integrate it into the multiscale permutation entropy, so as to achieve feature extraction at multiple scales. For example, Zhang et al. [13] introduced skewness in the process of applying multiscale entropy to extract feature information of multiscale entropy.

This paper introduces the concept of sample quantile on the basis of multiscale permutation entropy. Quantile divides the probability distribution range of a random variable into equal numerical points, regardless of the time dimension characteristics of the time series, and directly and effectively characterizes the characteristics of signal time series data fluctuations [19]. For the rolling bearing vibration signal, the entropy data of the multiscale arrangement have no obvious periodicity and change law. Therefore, the sample quantile feature can be used to deeply extract the internal features of the multiscale permutation entropy value.

Given $X_{1}, X_{2}, \ldots, X_{n}$ is a sample from a certain population, and define the order statistic as $X_{(1)} \leq X_{(2)} \leq, \ldots, X_{(n)}$. The $p$ quantile of this sample can be defined as

$$
\left\{\begin{array}{l}
m_{p}=X_{(k)}, \\
k=[n p]+1 .
\end{array}\right.
$$

In the formula, [•] represents the symbol of the rounding operation. $p$ is the quantile value, generally 0.25 , 0.5 , and 0.75 , for example, $p=0.25$ is often called 0.25 quantile.

The sample quantile has the advantages of simple calculation and small amount of calculation. And the sample quantile is a nonparametric statistic, which can accurately reflect the aggregation characteristics of the data at a certain quantile point in the absence of prior information about the overall distribution [20].

For multiscale permutation entropy, by introducing the concept of sample quantiles, it is possible to take advantage of the insensitive characteristics of sample quantiles to the influence of outliers, skip the influence of extreme values on the whole, and analyze permutation entropy under multiple scales. The value changes in the characteristics of internal agglomeration [21]. At the same time, the sample quantile is an asymptotic, unbiased, and consistent estimate of the overall quantile, so the multiscale permutation entropy feature extraction based on the sample quantile can better reflect the characteristics of the overall.

In summary, according to the multiscale permutation entropy calculation method, combined with the actual demand for vibration signal feature extraction, the feature extraction steps based on QMPE can be summarized as follows:

(1) Multiscale permutation entropy calculation: by solving the multiscale permutation entropy value of the obtained bearing vibration signal, multiple multiscale permutation entropy values under different scale factors are obtained. For example, by solving the multiscale permutation entropy of the scale factor $\tau=10$ on the vibration signal, a feature vector of dimension 10 composed of the multiscale permutation entropy can be obtained:

$$
\mathrm{MPE}=\left[\mathrm{PE}_{\tau=1}, \mathrm{PE}_{\tau=2}, \ldots, \mathrm{PE}_{\tau=10}\right],
$$

where $\mathrm{PE}_{\tau=i}$ represents the permutation entropy value under the scale factor $\tau=i$.

(2) Solve the sample quantile of the eigenvector in Step (1) to obtain the QMPE value. QMPE solves the sample quantile and obtains the changes in the internal agglomeration characteristics of the permutation entropy under multiple scales. Taking the quantile of 0.25 as an example, the QMPE of 0.25 is obtained as

$$
\text { 0.25QMPE }=\text { quantile (MPE, 0.25), }
$$

where quantile is the realization of the MATLAB function of the sample quantile, which represents the process of solving the sample quantile.

(3) According to the obtained QMPE eigenvalues, construct the rolling bearing performance degradation model to realize the rolling bearing degradation performance evaluation.

According to the calculation steps of QMPE, the calculation process of QMPE is simple, and the feature extraction of the rolling bearing can be realized by solving the three sample quantiles at different scales. Compared with time-domain and frequency-domain analysis methods, it not only avoids the redundancy between the information caused by the feature dimension which is too large [22] but also avoids the time-frequency-domain features between multiple components in the signal decomposition process. The dimension reduction work after extraction [23] simplifies the feature extraction process. The QMPE value can more accurately characterize the complexity of the sample signal, which can be used as an important indicator of the quantification of the degree of bearing failure. 


\section{Simulation Signal Analysis}

The simulation signals of different failure levels of typical bearing inner rings are used, and MATLAB is used for simulation analysis. Given the rolling bearing vibration signal is $X$, which is composed of two parts: fault signal $x_{i}$ and white noise $r$ :

$$
X=a x_{i}+b r
$$

In the formula, when $b$ is constant, the size of $a$ indicates the severity of the fault.

The setting parameters are as follows: sampling frequency $f_{s}=20,480 \mathrm{~Hz}$; signal length $L=102,400$; rotation frequency $f_{r}=15 \mathrm{~Hz}$; bearing inner ring fault characteristic frequency $f_{i}=90 \mathrm{~Hz}$; bearing structure resonance frequency $f_{\text {res }}=7000 \mathrm{~Hz}$. Define the exponential decay envelope as $E=\sum_{i} A e^{-\xi\left(t-i / f_{i}\right)}$, where the attenuation coefficient $\xi=0.0488$ and inner ring fault pulse-amplitude modulation function $A=\cos \left(2 \pi f_{r} t\right)$. When $0.5 \pi \leq 2 \pi f_{r} t \leq 1.5 \pi, A=0$. Get fault signals $x_{i}$ and $X$ :

$$
\begin{aligned}
& x_{i}=E \sin \left(2 \pi f_{\text {res }} t\right), \\
& X=a x_{i}+0.22 r,
\end{aligned}
$$

where $a$ is the failure degree coefficient; the larger the value, the more serious the failure degree. Take $a=1,5$, and 10, respectively, to indicate mild, moderate, and severe failures.

The 102,400-length signal is decomposed into 100 short signals with a length of 1024, the quantiles are, respectively, $0.25,0.5$, and 0.75 , and the QMPE values of these short signals are calculated. The results are shown in Figures 1-3.

The following conclusions can be drawn from Figures 1-3: the entropy values of QMPE under three different failure levels under $0.25,0.5$, and 0.75 are mild failure $>$ moderate failure $>$ severe failure. This is because the vibration of the rolling bearing is random vibration, and its vibration signal has a high degree of irregularity. The degree of irregularity is low, and the self-similarity is low, so the complexity of the sequence is high. The permutation entropy is a measure of the complexity of the time series. Therefore, the entropy value of the multiscale permutation under mild failures is larger at various scales. With the deepening of the fault degree, the regularity of the vibration signal increases, and the self-similarity increases, so the entropy value continues to decrease. Comparing the degree of discrimination in Figures 1 and 3, it can be seen that QMPE under 0.25 and 0.5 has a good degree of discrimination and can distinguish the three failure levels, while QMPE under 0.75 has a weak degree of discrimination, which proves the rationality of multiscale permutation entropy based on the quantile in reflecting the internal characteristics of the data. The simulation signal analysis shows that the signals of different failure degrees of the bearing inner ring can be quantitatively characterized by the QMPE value.

\section{Experiment Analysis}

In order to further verify the effectiveness of the proposed QMPE for the evaluation of the failure degree of rolling

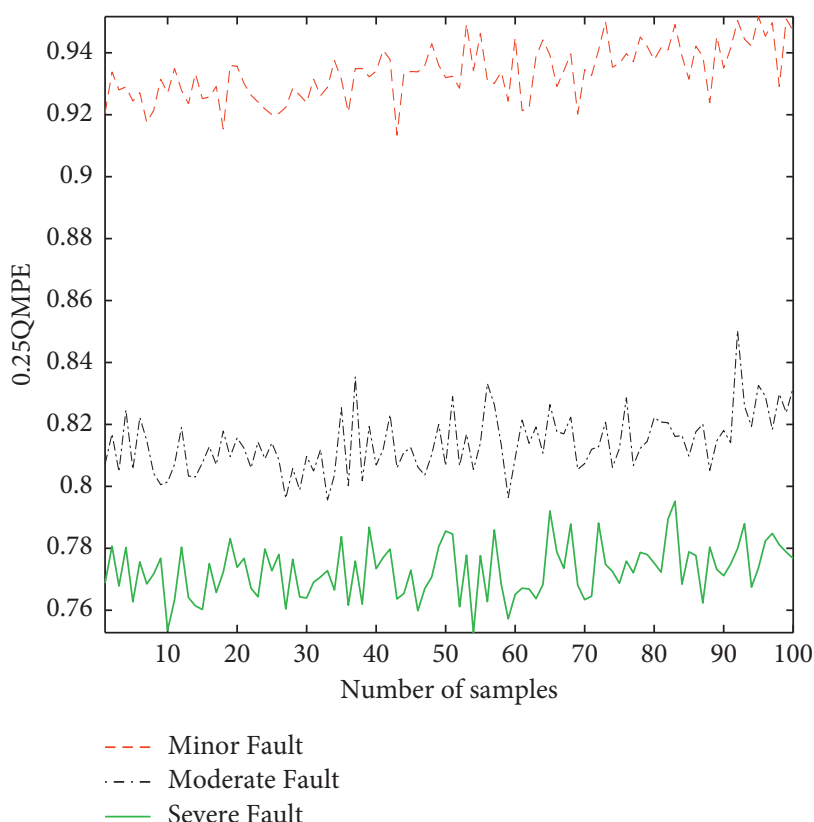

Figure 1: The 0.25 QMPE of signals derived from different fault severities.

bearing data, the following uses QMPE to realize the analysis of the measured signals.

5.1. Experimental Data. In order to compare with the existing literature, the experimental data selected in this paper come from the rolling bearing dataset of NASA. The data are provided by the research group of Professor Jay LEE of Cincinnati University and the American Intelligent Maintenance Center (IMS) [24]. The rolling bearing test bench is shown in Figure 4.

As shown in Figure 4, there are 4 double-row rolling bearings in the main shaft of the test bench; the model is Rexnord ZA-2115. Both ends of the bearing are fixed, and a radial load of $2721.6 \mathrm{~kg}$ is applied to the main shaft by a lever mechanism through bearings 2 and 3. During the experiment, each bearing is forced to be lubricated, and the lubrication condition is measured by the thermocouples equipped on the 4 bearing seats to realize real-time monitoring of the bearing outer ring temperature. The degree of failure of the gyro is judged by the magnetic plug arranged in the oil return pipe of the lubrication system. The magnetic plug collects metal abrasive particles in the lubricating fluid. When the number of metal particles reaches a certain level, it indicates that the bearing has suffered a serious failure, thus ending the experiment.

In order to compare the pros and cons of other fault state evaluation algorithms, the dataset used in this paper is consistent with other documents, and the second test data of this experiment are selected. The data are collected by the 6062E-type acquisition card of NI Company in the United States. The sampling frequency is $20 \mathrm{kHz}$, and each file contains 20,480 data points. The time interval between every two files is 10 minutes. The start time of data collection was 10:32:39, February 12, 2004, and the end time was 06:22:39, 


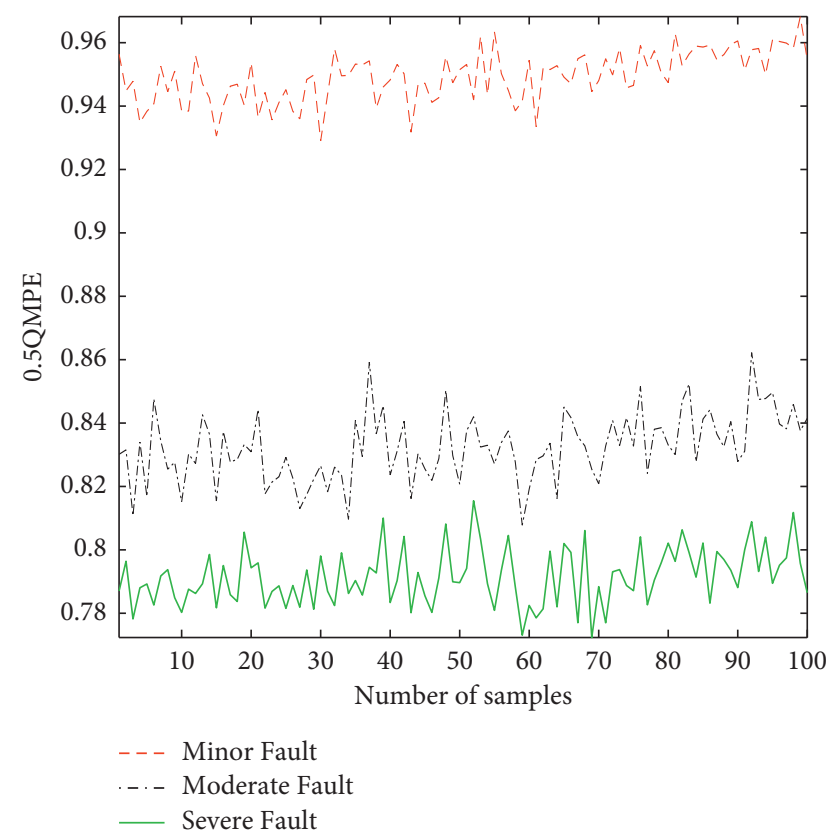

Figure 2: The 0.5 QMPE of signals derived from different fault severities.

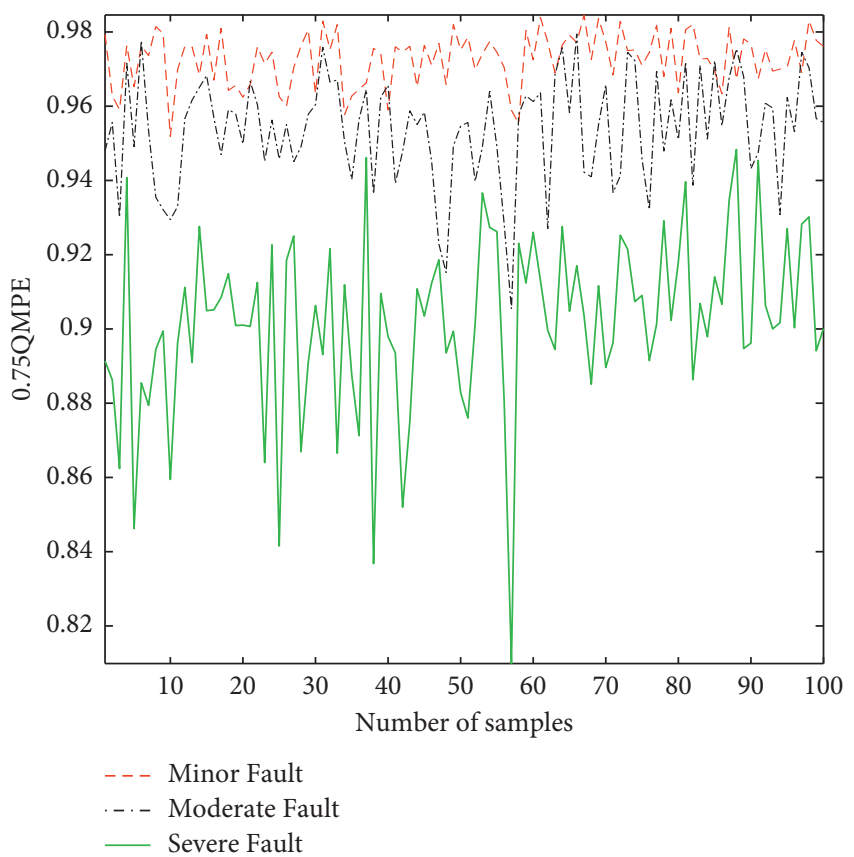

Figure 3: The 0.75 QMPE of signals derived from different fault severities.

February 19, 2004. A total of 984 files were collected, and the test lasted 163.8 hours. After the test, the outer ring of bearing 1 was severely worn.

5.2. Diagnostic Analysis. In order to verify the effectiveness of the QMPE algorithm in early fault diagnosis, this algorithm is used to analyze the original bearing vibration data. In terms of data processing, due to the large data length, the

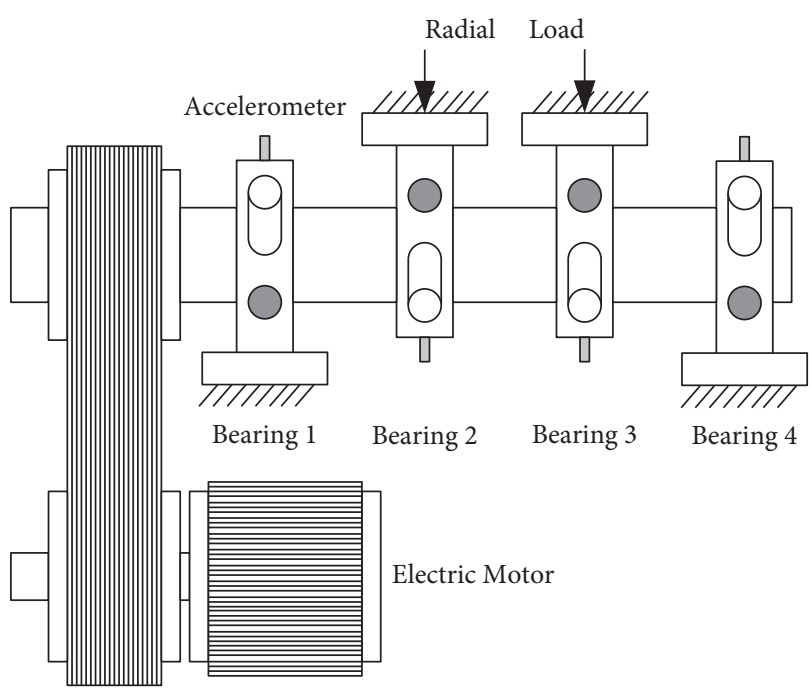

FIgURE 4: Schematic of the test.

method used in [9] and [10] is used to divide 20480 data points in each of the 984 files into 20 segments, each with 1024 data points. Calculate the QMPE for each segment separately, and finally, use the average of these 20 entropy values as the QMPE of the file data. As the bearing wears severely at the later stage of the data, the performance index has abrupt changes at this time, which is not convenient for drawing and display, so the first 942 files out of the 984 files are selected for display. In the calculation of QMPE, the embedding dimension $m=4$, the delay time $\tau=1$, and the scale factor is selected as 10. The QMPE under $0.25,0.5$, and 0.75 of the first 942 files is shown in Figures 5-7.

Take Figure 5 as an example for analysis, and extract the trend item of 0.25 QMPE in Figure 5 for easy observation. The result is shown in Figure 8.

As shown in Figure 8, the bearing belongs to the running-in period at the initial stage and is in a stable state after a short period of fluctuation. During normal operation, the bearing has been in a stable operation stage. Although the 0.25 QMPE value has a partial increase or decrease, the fluctuation range is not large, and this stage can be judged to be the normal operation period of the bearing.

After the 83rd hour of operation, the value of $0.25 \mathrm{QMPE}$ showed a linear downward trend. At this time, it indicates that the abnormality of the bearing begins to occur. At this time, it can be determined as an early fault of the bearing.

The bearing continued to work after the early failure. After a period of stability, the bearing showed a linear upward trend at $112.3 \mathrm{~h}$, indicating that the fault has occurred at this time. By analyzing the 0.25 QMPE value, it can be judged that the detection result of the early weak bearing failure is $83 \mathrm{~h}$.

Similarly, the trend term of QMPE in Figures 6 and 7 is extracted, and the results are shown in Figures 9 and 10.

As shown in Figures 9 and 10, the result of 0.5 QMPE analysis is that the early fault occurs at $82.5 \mathrm{~h}$, and the result of 0.75 QMPE analysis is that the early fault occurs at $82.33 \mathrm{~h}$. The feature vectors obtained through QMPE analysis can detect the weak changes of the early fault earlier. 


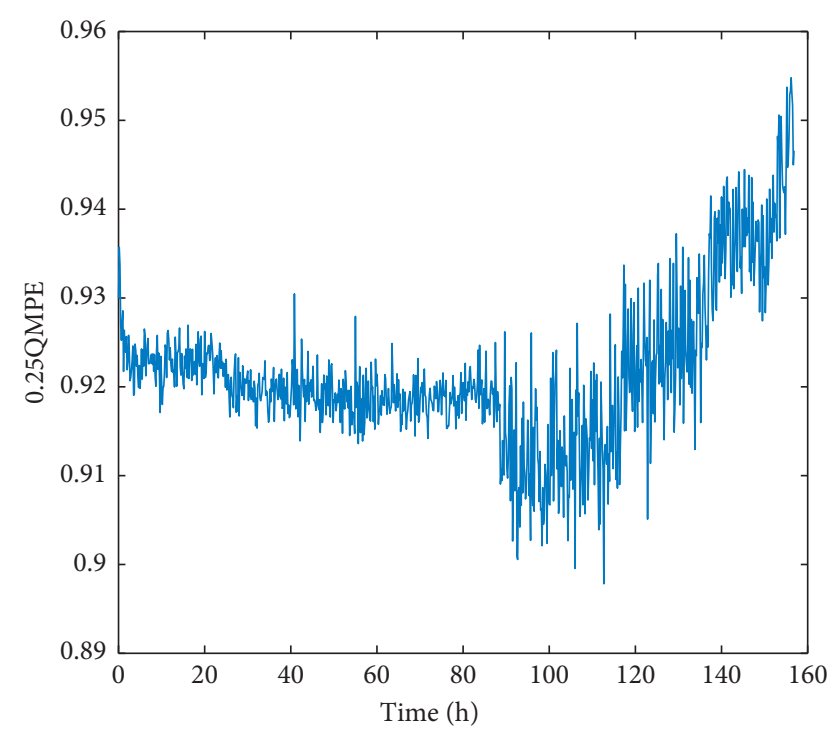

FIGURE 5: 0.25 QMPE of the experimental data.

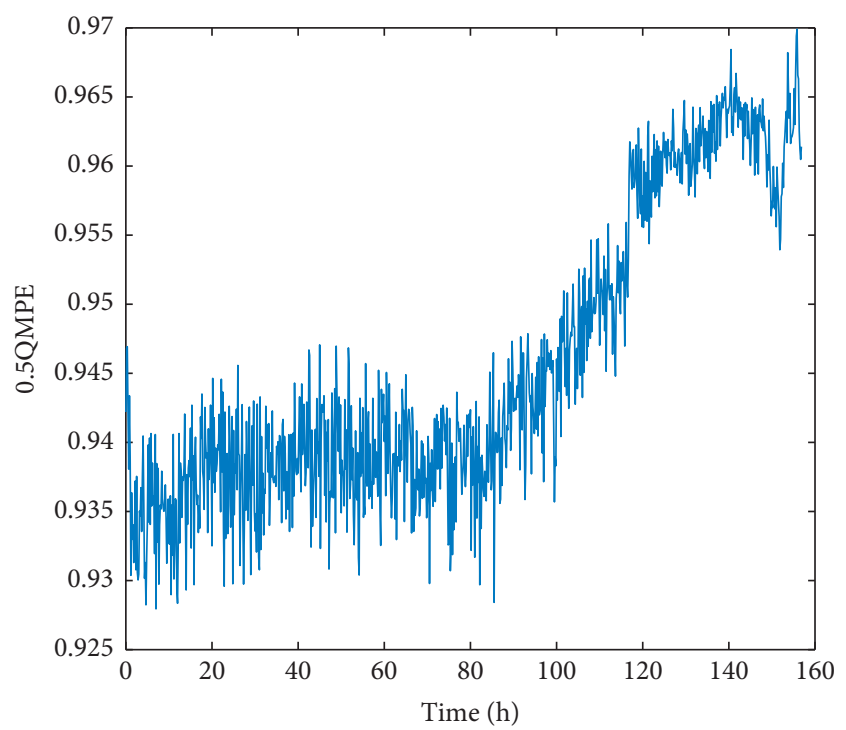

FIgURE 6: 0.5 QMPE of the experimental data.

5.3. Comparative Analysis. Feng et al. [9] used EMD to decompose rolling bearing signals and then used permutation entropy algorithm for analysis. The analysis data are the same dataset. During the calculation of permutation entropy, the embedding dimension $m=4$ and delay time $\tau=1$ are determined by the mutual information method and pseudo-nearest-neighbor method. The data preprocessing is to divide 20,480 data points into 20 segments, and the length of each segment is 1024 . The test data are the same and can be compared and analyzed. The results are shown in Figure 11.

The trend term of PE in Figure 11 is extracted for observation. The results are shown in Figure 12.

As shown in Figure 12, according to the analysis result of [9], the PE value jumps at $88.83 \mathrm{~h}$, so it is considered to be an early failure of the bearing; from $116.5 \mathrm{~h}$ to $118 \mathrm{~h}$, the

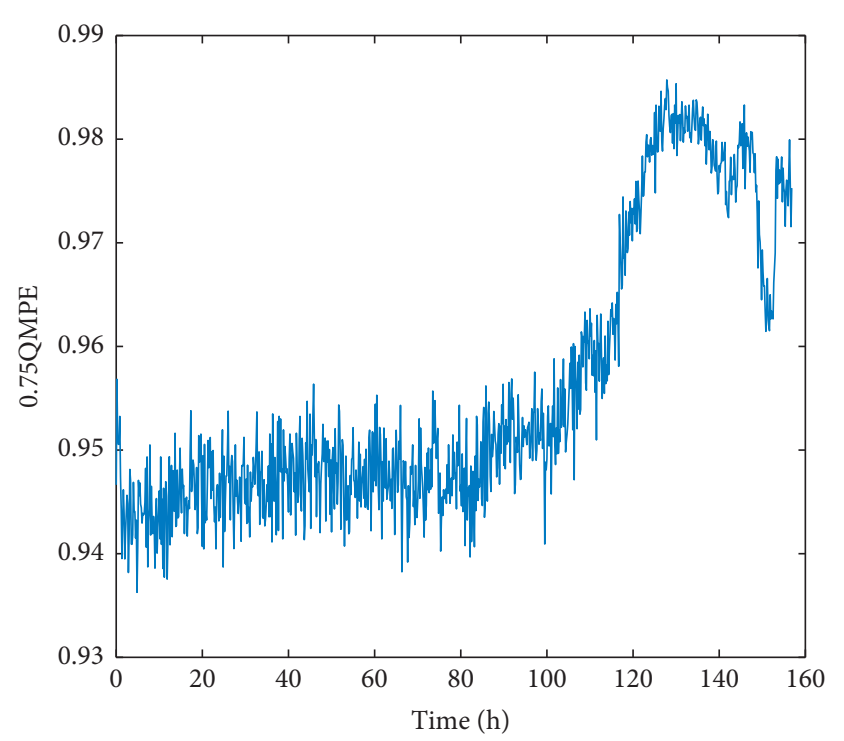

FIgURE 7: 0.75 QMPE of the experimental data.

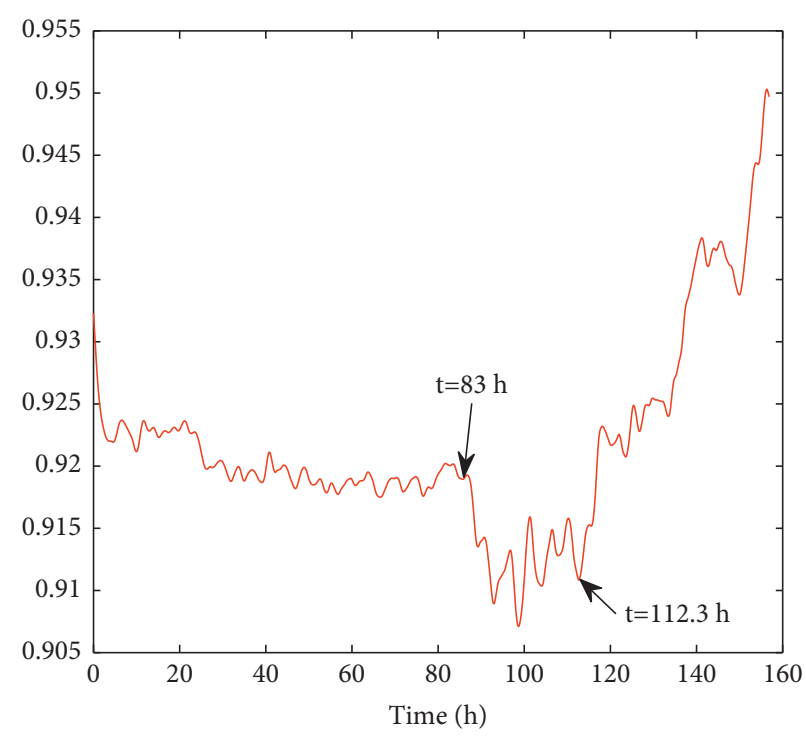

Figure 8: The trend of 0.25 QMPE.

permutation entropy curve drops significantly, the decline is relatively large, and the eigenvalue reaches the lowest. It is considered that the bearing failure has occurred at this time. In contrast, this article takes the result of $0.25 \mathrm{QMPE}$ as an example, the diagnosis result is that the early failure occurred at $83 \mathrm{~h}$, and the failure occurred at $112.3 \mathrm{~h}$. The diagnosis result is $5.83 \mathrm{~h}$ and $4.2 \mathrm{~h}$ earlier than [9].

Wavelet correlation permutation entropy in [10], wavelet entropy in [25], wavelet correlation characteristic scale entropy in [26], multifrequency scale fuzzy entropy in [12], and lifting wavelet packet singular spectrum entropy in [27] are selected for comparative analysis. The results are shown in Table 1.

It can be seen from Table 1 that the QMPE method proposed in this paper extracts features of rolling bearing vibration signals, which can effectively determine the early 


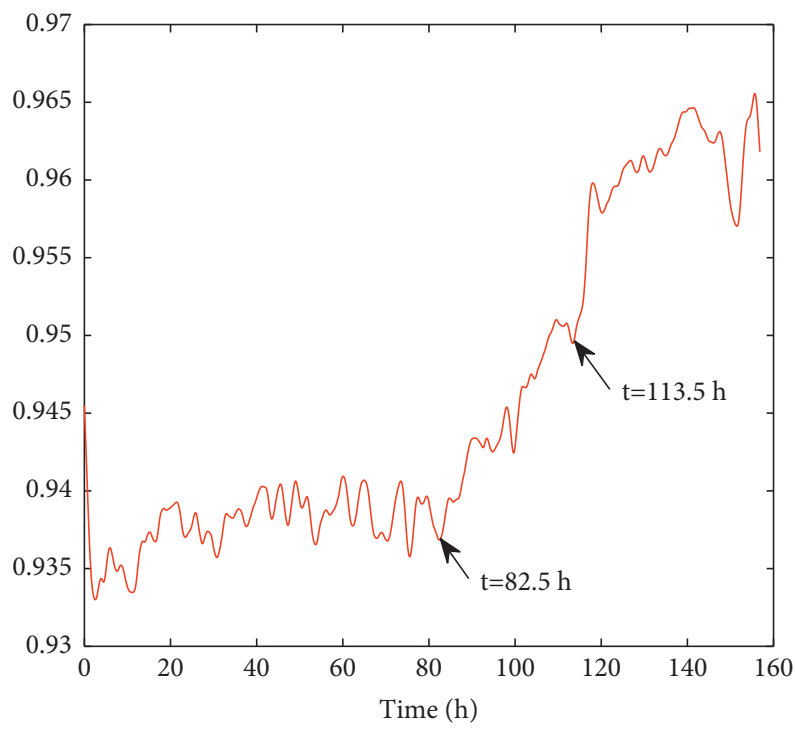

Figure 9: The trend of 0.5 QMPE.

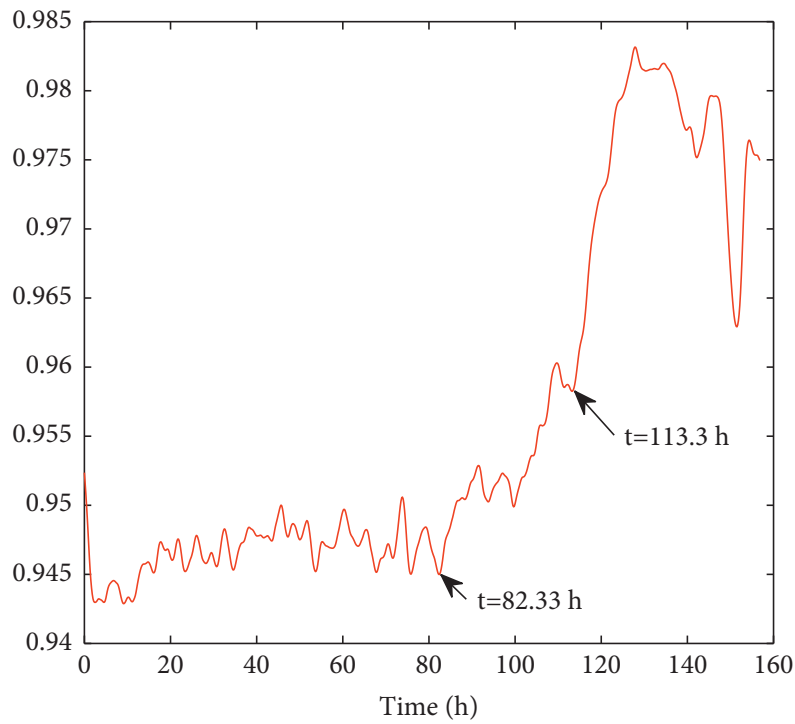

Figure 10: The trend of 0.75 QMPE.

weak fault signals of rolling bearings. Compared with other documents, it can advance the detection time of faults and is more suitable for early fault diagnosis.

The existing early fault diagnosis methods can generally reflect the whole life process of bearing operation. However, because the characteristic reflection of the signal is weak when the early minor failure of the bearing occurs and the signal information is easily submerged in the noise generated by the environment and the process in the actual use process, this brings difficulties to the early diagnosis of the 


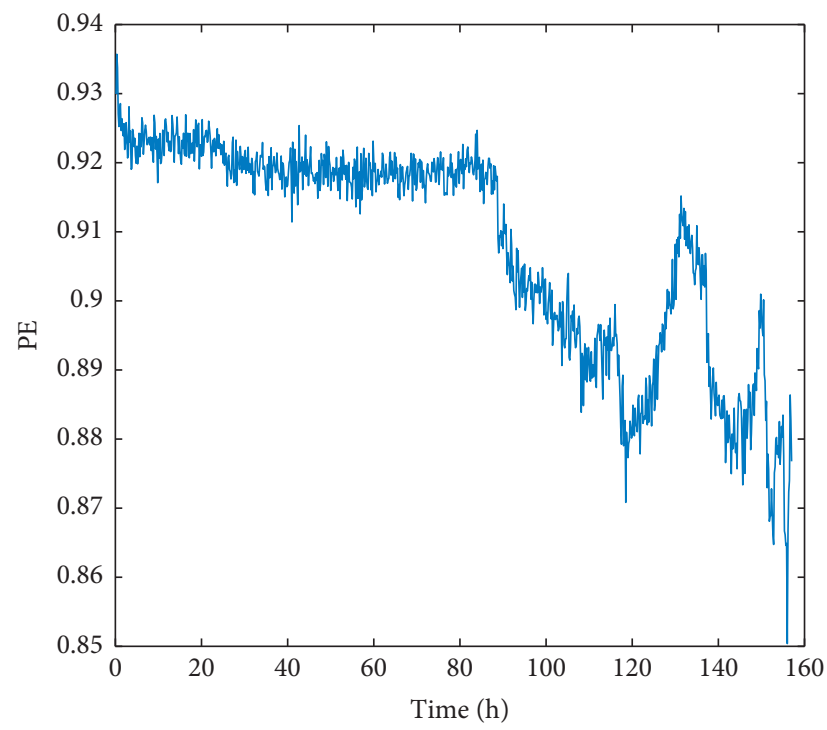

Figure 11: PE of the experimental data.

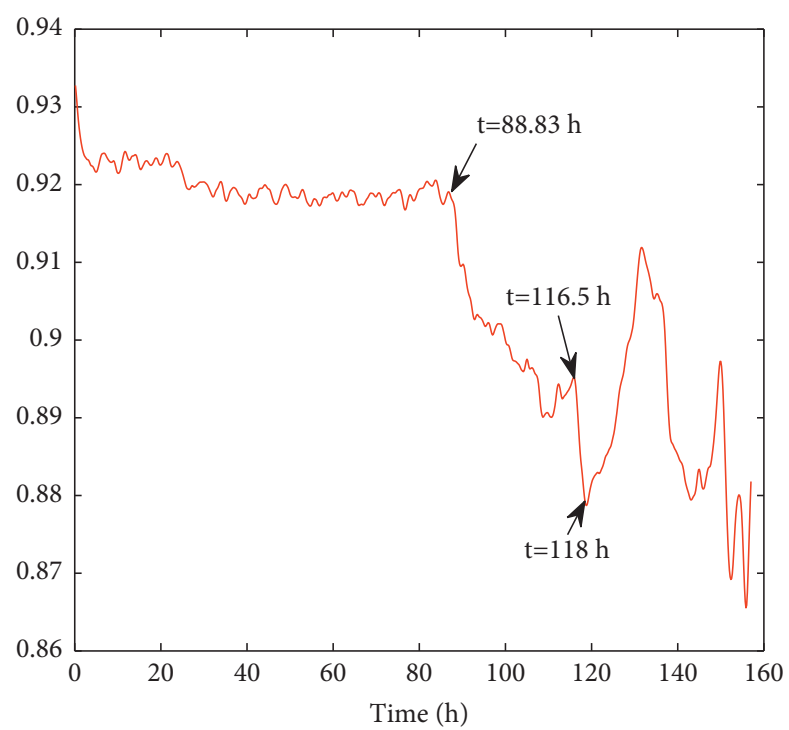

Figure 12: The trend of PE.

TABLE 1: Early fault diagnosis of different algorithms.

\begin{tabular}{lccc}
\hline Algorithm & $0.25 \mathrm{QMPE}$ & $0.5 \mathrm{QMPE}$ & $0.75 \mathrm{QMPE}$ \\
\hline Failure time & $83 \mathrm{~h}$ & $82.5 \mathrm{~h}$ & $82.33 \mathrm{~h}$ \\
Algorithm & Feng et al. [9] & Feng et al. [10] & Wang et al. [12] \\
Failure time & $88.83 \mathrm{~h}$ & $86.8 \mathrm{~h}$ & $96.66 \mathrm{~h}$ \\
Algorithm & Yin et al. [25] & Zeng et al. [26] & Xu [27] \\
Failure time & $96.7 \mathrm{~h}$ & $94.2 \mathrm{~h}$ & $88.83 \mathrm{~h}$ \\
\hline
\end{tabular}


bearing failure. Early diagnosis of faults in advance can leave sufficient early warning time for maintenance personnel, thereby improving equipment reliability.

\section{Conclusion}

This paper defines the sample quantile multiscale permutation entropy (QMPE), which is verified by the whole life test data of the rolling bearing. Through the QMPE feature extraction of the rolling bearing vibration signal, the early fault diagnosis of the rolling bearing is realized. Through experimental analysis and comparison with other literature studies, QMPE algorithm can effectively advance the fault detection time and maintain good consistency with the fault development trend. This article can draw the following conclusions:

(1) The permutation entropy algorithm can extract and amplify the small changes in the time series and accurately detect the sudden changes in the time series.

(2) The feature extraction of MPE based on the sample quantile can effectively extract the agglomeration situation within the data and improve the efficiency of fault extraction.

(3) Compared with other methods, the fault diagnosis method based on QMPE has simple calculation and can significantly improve the detection time of early faults. It is an effective fault detection method.

\section{Data Availability}

The experimental data selected in this paper come from the rolling bearing dataset of NASA. The data are provided by the research group of Professor Jay LEE of Cincinnati University and the American Intelligent Maintenance Center (IMS) [24].

\section{Conflicts of Interest}

The authors declare that they have no conflicts of interest.

\section{References}

[1] F. Wang, Y. Zhang, B. Zhang, and W. Su, "Application of wavelet packet sample entropy in the forecast of rolling element bearing fault trend," in Proceedings of the 2011 International Conference on Multimedia and Signal Processing, Guilin, China, May 2011.

[2] W. A. Smith and R. B. Randall, "Rolling element bearing diagnostics using the case Western Reserve University data: a benchmark study," Mechanical Systems and Signal Processing, vol. 64-65, pp. 100-131, 2015.

[3] X. Gu, S. Yang, Y. Liu, and R. Hao, "Rolling element bearing faults diagnosis based on kurtogram and frequency domain correlated kurtosis," Measurement Science and Technology, vol. 27, no. 12, pp. 1-15, 2016.

[4] P. Gangsar and R. Tiwari, "Multiclass fault taxonomy in rolling bearings at interpolated and extrapolated speeds based on time domain vibration data by SVM algorithms," Journal of Failure Analysis and Prevention, vol. 14, no. 6, pp. 826-837, 2014.

[5] D. Xu, J. Ge, Y. Wang, and J. Shao, "Prediction of rolling bearing performance degradation based on manifold learning and M-KH-SVR," Journal of Vibration Engineering, vol. 31, no. 5, pp. 892-901, 2018.

[6] F. Wang, Research on the Degradation Trend and Residual Life Prediction Method of Rolling Bearing Based on ELM, Anhui University of Technology, Ma'anshan, Anhui, China, 2018.

[7] J. Zhou, Q. Xu, L. Zhang, and P. Li, "Assessment method of rolling bearing performance degradation based on wavelet packet singular spectral entropy and SVDD," Mechanical Science and Technology for Aerospace Engineering, vol. 35, no. 12, pp. 1882-1887, 2016.

[8] J. Zhou, Q. Xu, L. Zhang, and H. Li, "Rolling bearing performance degradation assessment based on the wavelet packet Tsallis entropy and FCM," Journal of Mechanical Transmission, vol. 40, no. 5, pp. 110-115, 2016.

[9] F. Feng, G. Rao, L. Zhang, and A. Si, "Research on abnormality detection method for bearings based on EMD and permutation entropy," Bearing, no. 2, pp. 53-56, 2013.

[10] F. Feng, A. Si, and G. Rao, "Early fault diagnosis technology for bearing based on wavelet correlation permutation entropy," Journal of Mechanical Engineering, vol. 48, no. 13, pp. 73-79, 2012.

[11] C. Bandt, Permutation Entropy and Order Patterns in Long Time Series, Springer International Publishing, Berlin, Germany, 2016.

[12] F. Wang, W. Li, J. Zheng, and P. Zheng, "Prediction of remaining life of rolling bearing based on multi-frequency scale fuzzy entropy and ELM," Noise and Vibration Control, vol. 38, no. 1, pp. 188-192, 2018.

[13] L. Zhang, W. Huang, and G. Xiong, "Assess of rolling element bearing fault severity using multi-scale entropy," Journal of Vibration and Shock, vol. 33, no. 9, pp. 185-189, 2014.

[14] R. Yan, Y. Liu, and R. X. Gao, "Permutation entropy: a nonlinear statistical measure for status characterization of rotary machines," Mechanical Systems and Signal Processing, vol. 29, pp. 474-484, 2012.

[15] B. Christoph and P. Bernd, "Permutation entropy: a natural complexity measure for time series," Physical Review Letters, vol. 88, no. 17, Article ID 174102, 2002.

[16] J. Zheng, J. Cheng, and Y. Yang, "A rolling bearing fault diagnosis approach based on multiscale entropy," Journal of Hunan University, vol. 39, no. 5, pp. 38-41, 2012.

[17] J. Ren, G. Sun, B. Chen, and M. Luo, "Multi-scale permutation entropy based on-line milling chatter detection method," Journal of Mechanical Engineering, vol. 51, no. 9, pp. 206-212, 2015.

[18] W. Yao, T.-B. Liu, J.-F. Dai, and J. Wang, "Multiscale permutation entropy analysis of electroencephalogram," Acta Physica Sinica, vol. 63, no. 7, pp. 427-433, 2014.

[19] M. I. Salazar-Alvarez, C. Temblador-Pérez, W. J. Conover, V. G. Tercero-Gómez, A. E. Cordero-Franco, and M. G. Beruvides, "Regressing sample quantiles to perform nonparametric capability analysis," International Journal of Advanced Manufacturing Technology, vol. 86, no. 5-8, pp. 1347-1356, 2016.

[20] C. Jentsch and A. Leucht, "Bootstrapping sample quantiles of discrete data," Annals of the Institute of Statistical Mathematics, vol. 68, no. 3, pp. 491-539, 2016.

[21] J. Li, B. Jin, X. Qiang, and X. Liu, "Fault states feature extraction and experimental study for airborne fuel pumps 
based on sample quantile," Acta Aeronautica et Astronautica Sinica, vol. 37, no. 9, pp. 2851-2863, 2016.

[22] H. Dai, A. Xu, W. Li, and W. Sun, "Fault diagnosis of rolling bearings in mixed domain based on WMRMR," Journal of Vibration and Shock, vol. 34, no. 19, pp. 57-61, 2015.

[23] J. Dai, Multiscale Entropy Theory Based Fault Diagnosis of Rolling Bearing, Anhui University of Technology, Ma'anshan, Anhui, China, 2017.

[24] J. Lee, H. Qiu, G. Yu, J. Lin, and Rexnord Technical Services, IMS, University of Cincinnati, "Bearing Data Set," NASA Ames Prognostics Data Repository, NASA Ames Research Center, Mountain View, CA, USA, 2007, (http://ti.arc.nasa. gov/project/prognostic-data-repository).

[25] X. Yin, Y. He, Z. Peng, and F. Chu, "Study on wavelet entropy and its applications in trend analysis," Journal of Vibration Engineering, vol. 17, no. 2, pp. 165-169, 2004.

[26] Q. Zeng, J. Qiu, and G. Liu, "Application of wavelet correlation feature scale entropy and hidden semi-markov models to equipment degradation state recognition," Chinese Journal of Mechanical Engineering, vol. 44, no. 11, pp. 236-241, 2008.

[27] Q. Xu, Rolling Bearing Performance Degradation Assessment Based on Support Vector Data Description, East China Jiaotong University, Nanchang, China, 2015. 